\title{
Design and Implementation of Low Cost Manipulator Robot for Underwater Object Grasping Process
}

\author{
M. Manoj Prabhakar, K. Maynadi, M .S. Abilash, N.Rajini, K. Subash
}

\begin{abstract}
Various developments in the technologies over the industrial sectors, Robots are implemented to perform the several processes like picking and placing objects by using a human hand like structures. These robotic manipulators are mainly employed in the areas which are radioactive or hazardous or the place not accessible by the humans. It uses both direct pneumatics and inverse pneumatics. Underwater object or material grasping is a challenging task in these days. Here we are going to design and implement the robot manipulator at low-cost for object grasping process. The 3 DOF underwater robot manipulator can be developed by using the servo motors embedded in a mechanical setup. A camera based system is used to identify the objects to be grasped. These whole processes can be controlled by using Raspberry pi 3 as a central processor. The movement of the robot manipulator is controlled by using position control joystick through Internet of Things (IoT).
\end{abstract}

Keywords: Robot manipulator, direct pneumatic, inverse pneumatics.

\section{INTRODUCTION}

Some components, such as multi-leveled equations, graphics, and tables are not prescribed, although the various table text styles are provided. The formatter will need to create The robotic manipulators are not only employed for the application in the industrial sectors but also it is employed for the deepwater investigation. Nowadays mining the deep sea resources like gas or oil become the important trend for the offshore industries [1-2]. It is highly expensive in these days. A cost effective system is the only solution for this problem. The robotic manipulators provide the solution for the problem. It will be used along with other robots used for the underwater process [3]. The operating process of the manipulator robots can be remotely operated vehicles

Revised Manuscript Received on December 05, 2019.

* Correspondence Author

M. Manoj Prabhakar, School of Automotive and Mechanical Engineering. Kalasalingam Academy of Research and Education, Krishnankoil-626 126, India. Email: m.manojprabhakar@klu.ac.in

K.Maynadi, School of Automotive and Mechanical Engineering. Kalasalingam Academy of Research and Education, Krishnankoil-626 126, India. Email: k.mayandi@klu.ac.in

M. S. Abilash, School of Automotive and Mechanical Engineering. Kalasalingam Academy of Research and Education, Krishnankoil-626 126, India. Email: abilash.nivas@gmail.com

N.Rajini, School of Automotive and Mechanical Engineering. Kalasalingam Academy of Research and Education, Krishnankoil-626 126 , India. Email: n.rajini@klu.ac.in

K. Subash, School of Automotive and Mechanical Engineering. Kalasalingam Academy of Research and Education, Krishnankoil-626 126, India. Email:
(ROV's) where the controlling of the manipulator robot can be done manually and it may be an Automatic Underwater Vehicles (AUV's) where the robotic manipulator can able operate automatically [4]. These employed robotic manipulator vehicles have used for the various applications like undersea mining and examination operation, in searching for the valuable objects Fig 1 which have been lost in the sea, laying and tracking the cables in the under water. These manipulator robots which are interfaced with the processor can be easily controlled by the user or operator very efficiently either by auto or manually by using position controlled joystick through IoT [5-6]. This process can be achieved by taking manipulator robot as the configuration of master and slave. Operator controls the slave arm with the movement of the master arm. Operation of the system is fully based on the one or more camera in the vehicle system ROV's and AUV's

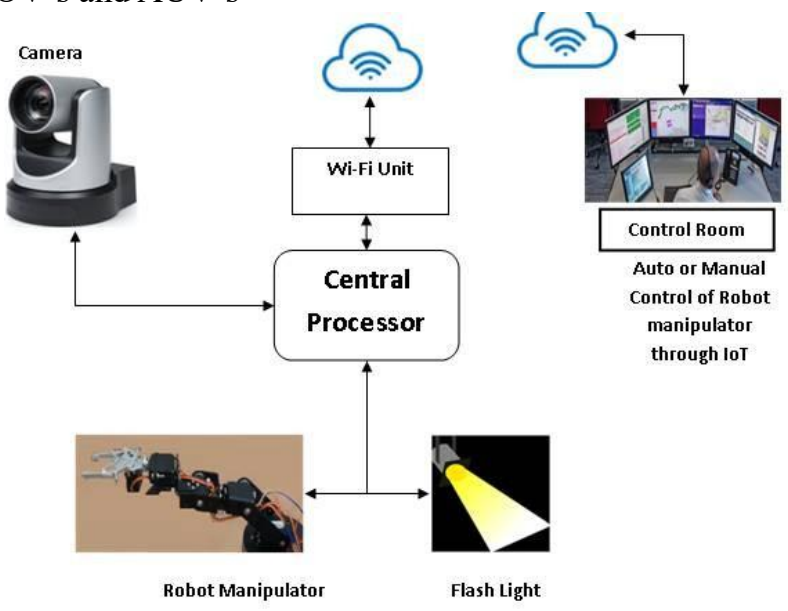

Figure 1. System architecture for implementation of a low cost manipulator robot for underwater object grasping process

\section{EXPERIMENTAL METHODOLOGY}

The primary objective of our proposed system is to develop the low cost manipulator robot for the underwater grasping process. The main arm and the auxiliary arm of our robot setup are made with the servo motor. This robotic manipulator setup is interfaced with the raspberry pi3. It is acts as a central processor here.

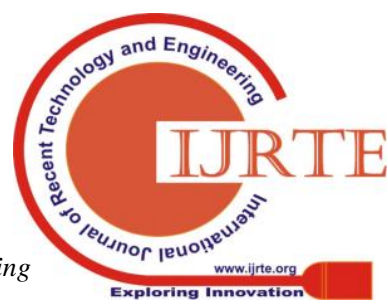




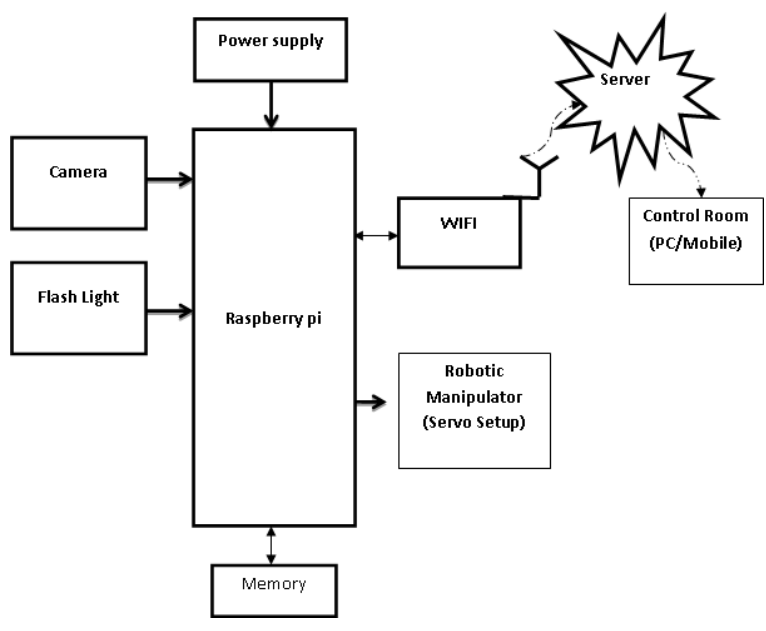

Figure 2. Block Diagram for implementation of a low cost manipulator robot for underwater object grasping process

Underwater Manipulator robot is placed in the vehicle which can be remotely controlled or it can operate automatically. The movement of the vehicle is mainly based on the camera system. Flash light is also interfaced with the central processor. Figure 2 shows the block diagram for low cost implementation of an underwater manipulator robot for grasping the objects from the underwater.

\subsection{Hardware Components}

\subsubsection{Camera}

Camera acts as a vision for our proposed system. Camera is interfaced with the central processor. It is used to recognize the object to be identified. The operator can choose the object for finding. Once the object to be searched is chosen then the robotic vehicles approach the object.

\subsubsection{Flash light}

Flash light is employed in our system in order to support the vision of the robotic manipulator in the deep water.

\subsubsection{Robotic manipulator}

The system is employed with 3 DOF robotic manipulator. The setup of the robotic manipulator is normally used for the master slave control. This can be achieved by using the robotic setup as the position feedback. The setups of this system have axes and claws for grasping. Figure 3 shows the model representation of the robotic hand manipulator employed for grasping the valuable objects in the underwater. It consists of shoulder, elbow jaw and wrist. The slave arm can be controlled by receiving the commands from the master arm. The wrist arm is equipped with camera for the visual applications. An additional camera is placed for the recognition of the object or the scene where the robotic manipulators are passing through. The main trouble may arise in a dynamic

environment where the robotic manipulator is not speedy for the task of grasping the objects. This whole movement can be controlled through IoT in the effective manner

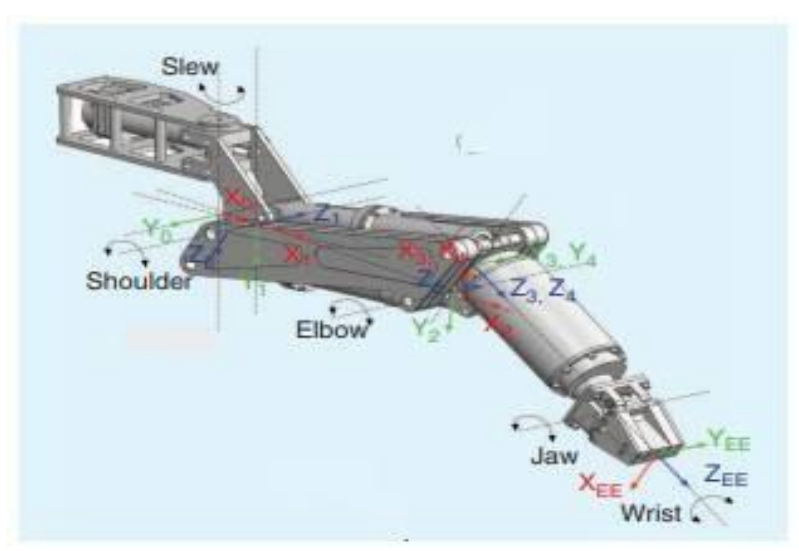

FigURE 3.MODEL DIAGRAM OF THE ROBOTIC MANIPULATOR

\section{Methodology}

Then the camera estimates the position of the object. Analysis of kinematics comprises of direct kinematics and inverse kinematics which is the most important one for studying the robot manipulator. The calculation for the inverse kinematics is complex when compared with forward kinematics. Figure 4 shows the architecture of control approach for kinematics. The analysis of direct kinematics in the manipulator robot is carried out for the finding the position and the orientation of the end object to be grasped from the manipulator base. The inverse kinematics is employed for finding the angle of the arm in robotic manipulator

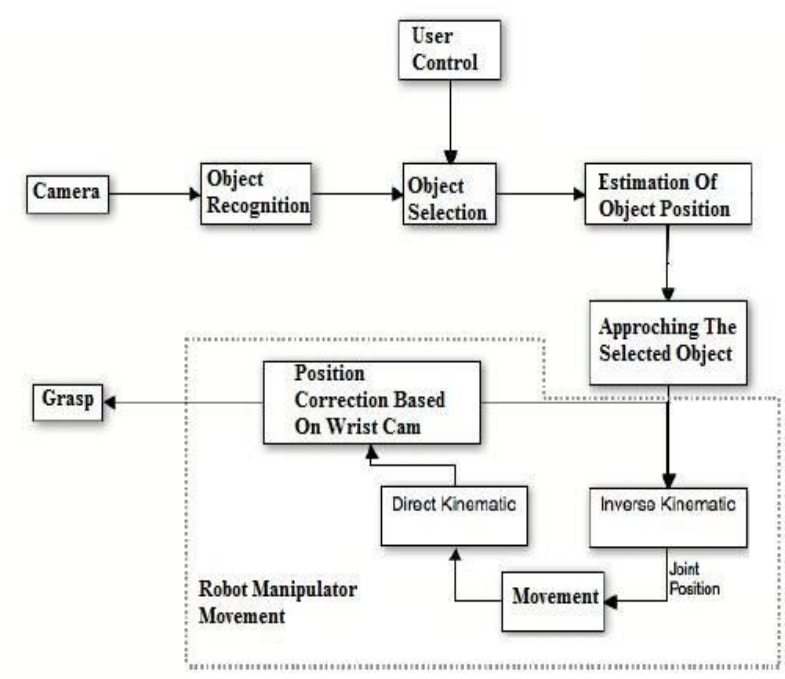

Figure 4.Architectureof the control approach of kinematics

Published By: 


\section{RESULTS AND DISCUSSION}

Our proposed robotic manipulator which is placed in the ROV's and AUV's can be controlled through the IoT. The system can have the capability of finding and grasping the objects which are in the stationary position. This implies that the objects to be grasped should be fixed in the place without any outer disturbances. Figure 5 shows the stationary objects which are grasped by the
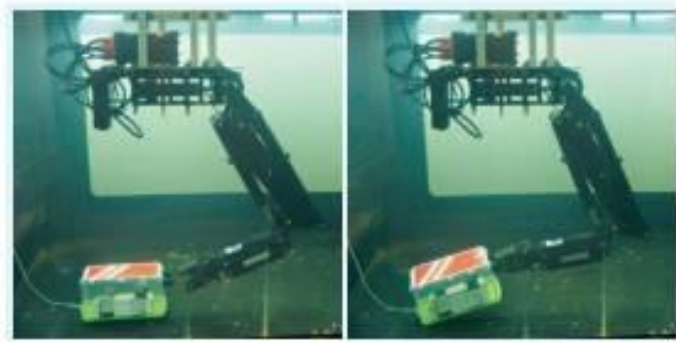

robotmanipulator

Figure 5.Stationary Object Grasped by Robot Manipulator

\section{CONCLUSION}

This article proposes the design and implementation of a low cost manipulator robot for underwater grasping of objects. The joystick with three directional position controls is used for commanding the joint angle for the manipulator operations. This process can be controlled by the operator through IoT. In our proposed work only the objects which are in stationary position can be grasped and moved to the required place. In our future work, a low cost manipulator for grasping the objects over the dynamic changes in their positions can be designed and developed for using it in various situation as feasible and also the capabilities of the existing ROV's and AUV's can be enhanced.

\section{CONFLICT OF INTEREST}

The authors declare no conflict of interest

\section{ACKNOWLEDGMENT}

Authors would like to acknowledge the support of Kalasalingam Academy of Research and Education, KARE, Department of Mechanical Engineering and School of Automotive and Mechanical Engineering (SAME).

\section{REFERENCES}

1. J.R. Stanley, The Station Keep TM Function: Dynamic positioning for remotely operated vehicles, In Proc. Int. Conf. Underwater Intervention, 2005.

2. K. Ioi and K. Itoh, "Modeling and simulation of an underwater manipulator", Advanced Robotics, 4(4), 303-317, 1989.

3. I. Schjolberg and T. I. Fossen, "Modeling and control of underwater vehicle-manipulator systems", In Proc. rd Conf. on Marine Craft maneuvering and control, 1994.

4. C.C. De Wit, E.O. Dia. and M. Perrier, "Robust nonlinear control of an underwater vehicle/manipulator system with composite dynamics", In Robotics and Automation, 1998. Proceedings. IEEE International Conference, 452-457, 1998

5. N. Sakagami and S. Kawamura, "Analysis on dynamics of 3-dof underwater robot manipulators based on time- scale transformation", In Proc. of 2002 Japan-USA symposium on Flexible Automation, 1139-1145, 2002.

6. S. R. Pandian, "Model-based sliding mode control of underwater robot manipulators", International Journal of Off shore and Polar Engineering, 16(03), 2006.

\section{AUTHORS PROFILE}

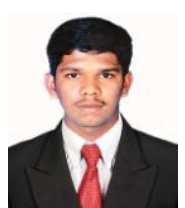

M. Manoj Prabhakar has received master degree in CAD/CAM from Kumaraguru College of Engineering \& Technology, Coimbatore, India. He is currently as the Assistant Professor at Kalasalingam Academy of Research and Education in the Department of Mechanical Engineering.. He had 7 years of Academic Experience

Second Author profile which contains their education details, their publications, research work, membership, achievements, with photo that will be maximum 200-400 words.

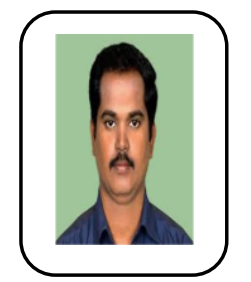

Dr.K.Mayandi received his B.E. (2007) in Mechanica Engineering and M.E. (2011) in Engineering Design from Anna University, Chennai and obtained the Ph.D (2016) from Kalasalingam University, Krishnankoil. $\mathrm{He}$ is working as an Associate Professor in the School of Automotive and Mechanical Engineering at Kalasalingam Academy of Research and Education, Krishnankoil, Madurai, India. His research interest on characterization of natural fibres and utilized the natural fibres as reinforcement with polymer composites materials for biodegradable applications. Recently focuses the research on fracture analysis of layered polymer composites materials and also involves the works on fabrication and testing of filament winding polymer composites tube for medium load applications areas. He has published 17 international journals and 2 book chapters published in Elsevier and Wiley publications.



M.S. Abilash has received master degree in CAD/CAM from Kumaraguru College of Engineering \& Technology, Coimbatore, India. He is currently as the Research Scholar at Kalasalingam Academy of Research and Education in the Department of Mechanical Engineering.. He had 7 years of Academic Experience



Dr.N.Rajini is working as Professor in the department of Mechanical Engineering in Kalasalingam Academy of Research and Education since 2004. He completed his Ph.D in 2013 from Kalasalingam University. He completed his post graduation in 2000. His area of interest is composite materials. He has international collaborative projects and researches. He published more than $150 \mathrm{SCI}$ and SCI-Expended articles.

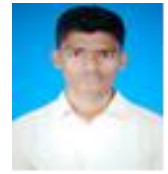

K. Subash, is the Student at Kalasalingam Academy of Research and Education in the Department of Mechanical Engineering 